\title{
A nonextensive entropy path to probability distributions in solar wind turbulence
}

\author{
M. P. Leubner ${ }^{1,2}$ and Z. Vörös ${ }^{1}$ \\ ${ }^{1}$ Space Research Institute, Austrian Academy of Sciences, Graz, Austria \\ ${ }^{2}$ Institute for Astrophysics, University of Innsbruck, Innsbruck, Austria \\ Received: 16 September 2004 - Accepted: 29 November 2004 - Published: 1 February 2005 \\ Part of Special Issue "Nonlinear processes in solar-terrestrial physics and dynamics of Earth-Ocean-System"
}

\begin{abstract}
The observed scale dependence of the probability distributions of the differences of characteristic solar wind variables is analyzed. Intermittency of the turbulent fluctuations at small-scale spatial separations is accompanied by strongly non-Gaussian distributions that turn into a normal distribution for large-scale separation. Conventional theoretical models are subject to insufficient physical justification since nonlocality in turbulence should be based on long-range interactions, provided recently by the bi-kappa distribution in the context of nonextensive thermostatistics. Observed WIND and ACE probability distributions are accurately reproduced for different time lags by the one-parameter bi-kappa functional, a core-halo convolution, where kappa measures the degree of nonlocality or nonextensivity in the system. Gradual decoupling is obtained by enhancing the spatial separation scale corresponding to increasing kappa values, where a Gaussian is approached for infinite kappa. Consequently, long-range interactions introduced on the fundamental level of entropy generalization, are able to provide physically the source of the observed scale dependence of the turbulent fluctuations in the intermittent interplanetary medium.
\end{abstract}

\section{Introduction}

The analysis of probability distribution functions (PDFs) is of considerable interest to study intermittency and multiscale statistical properties in fully developed turbulence of the solar wind plasma and interplanetary magnetic field fluctuations. Classical statistical theory provides the phase space distribution from ideal MHD invariants, introduced by Matthaeus and Goldstein (1982) to characterize spectral properties of incompressible solar wind fluctuations. The existence of multifractal structures in the fluctuations of the magnetic field strength and the plasma parameters as

Correspondence to: M. P. Leubner

(manfred.leubner@oeaw.ac.at) temperature, density and velocity of the interplanetary magnetofluid was confirmed (Burlaga, 1991, 1992), followed by analyses of non-Gaussian PDFs, related intermittency and the fractal scaling of the solar wind MHD fluctuations by Marsch and Tu (1994, 1997). The fractal/multifractal nature of certain solar wind and magnetospheric key parameters, however, remains a subject of recent investigations (Hnat et al., 2003). Interplanetary observations were used to establish the differences between fluid and MHD turbulence (Carbone et al., 1995) and the Castaing distribution (Castaing et al., 1990; Castaing and Dubrulle, 1995; Castaing, 1996) was widely considered in favor of models of the non-Gaussian cascade character of the intermittency in turbulent flows (Consolini and De Michelis, 1998; Guowei et al., 1998; Sorriso-Valvo et al., 1999; Schmitt and Marsan, 2001). In addition to other solar wind parameters as velocity and density, the non-Gaussian and intermittent properties of the interplanetary magnetic field fluctuations have shown to represent a new set of intermittency parameters controlling the energy input rate into the magnetosphere (Vörös et al., 2002; Vörös and Jankovičová, 2002). Multi-scale intermittency and anisotropy effects appear to be decisive factors in near-Earth magnetotail dynamics as well (Vörös, 2003, 2004). In particular, based on WIND, ACE and Voyager observations Burlaga et al. (2002); Burlaga (2002) (see also references therein) provide a comprehensive study of solar wind multi-scale statistical properties of speed fluctuations at $1 \mathrm{AU}$ and $60 \mathrm{AU}$, respectively, verifying that the PDFs at small scales have a shape that is characteristic for intermittent turbulence with relatively large skewness and kurtosis and approach a Gaussian for scales $\tau \sim 10$ days or $\tau \sim 24 \mathrm{~h}$, respectively, evaluated by Sorriso-Valvo et al. (1999).

The observed leptokurtic distributions in the solar wind turbulence are well represented by the empirical Castaing model (Castaing et al., 1990), a convolution of Gaussians as PDFs of small-scale differences $\delta X(\tau)=X(t+\tau)-X(t)$, where $X(t)$ denotes any characteristic solar wind variable at time $t$ and $\tau$ is the time lag, characterizing the spatial separation scale. The log-normal distribution of variances 
through the inertial scales ensures an excellent fit with the observed PDFs. On the other hand the global leptokurtic non-Gaussian shape of the increment PDFs requires theoretically a corresponding unique global distribution function. This condition can be formulated on a general level by considering the basic feature of turbulent flows, i.e. multi-scale coupling or nonlocality in physical or in Fourier space, where nonlocality appears due to the presence of long-range forces. In the simple case of turbulent cascades, the nonlinear term in the Navier-Stokes equations re-distributes energy among the Fourier modes. In general, however, the presence of the long-range forces in turbulence imply direct nonlocal interactions between large scales and small scales, since the relation between them is not local in space and time but functional. This indicates that small-scale fluctuations in each time/space point depend on the large scale motions in the whole time/space domain and vice versa (Tsinober, 2001). Counting for long-range interactions is a particular feature of nonextensive systems and available from pseudo-additive entropy generalization.

The classical Boltzmann-Gibbs extensive thermo-statistics constitutes a powerful tool when microscopic interactions and memory are short ranged and the environment is an Euclidean space-time, a continuous and differentiable manifold. However, in the present situation we are dealing with astrophysical systems, generally subject to spatial or temporal long-range interactions evolving in a nonEuclidean, for instance multi-fractal space-time that makes their behavior nonextensive. A suitable generalization of the Boltzmann-Gibbs-Shannon entropy for statistical equilibrium was first proposed by Renyi (1955) and subsequently by Tsallis (1988), preserving the usual properties of positivity, equiprobability and irreversibility, but suitably extending the standard extensivity or additivity to nonextensivity. The main theorems of the classical Maxwell-Boltzmann statistics admit profound generalizations within nonextensive statistics (sometimes referred to q-statistics where $q$ characterizes the degree of nonextensivity of the system) wherefore a variety of subsequent analyses were devoted to clarify the mathematical and physical consequences of pseudo-additivity, for an early review see e.g. Tsallis (1995).

Space physicists recognized four decades ago from in situ satellite observations frequently the existence of high-energy tail velocity space distribution of electrons and ions in space plasmas. The family of $\kappa$-distributions, a power-law in particle speed, was introduced on phenomenological grounds to model these suprathermal structures, where $\kappa$ assumes only positive values. In continuation, significant progress was provided by Treumann (1999a,b) who developed a kinetic theory, demonstrating that power-law velocity space distributions are a particular thermodynamic equilibrium state where the associated entropy resembles Tsallis' nonextensive entropy definition. One of the three free parameters of the theory, the chemical potential of the $\kappa$-gas, is associated with a $\kappa$-modified quantum density of the system and the classical Boltzmann entropy is recovered for $\kappa=\infty$. Relating the parameters $q$ and $\kappa$ by an elementary transformation Leubner
(2002) provided the missing link between nonextensive distributions and $\kappa$-functions favored in space plasma physics, leading to the required theoretical justification for the use of $\kappa$-distributions from fundamental physics. Since the parameter $\kappa$, a measure of the degree of nonextensivity of the system, is not restricted to positive values in the nonextensive context, the commonly observed core-halo twin character of the interplanetary electron and ion velocity space distributions was verified theoretically upon generalization to a bikappa distribution, subject to a less pronounced core along with extended tails as compared to a Maxwellian (Leubner, 2004a,b).

Recently, the PDF of the Tsallis ensemble was linked to the analysis of fully developed turbulence providing a relation between the nonextensive parameter $q$ and the intermittency exponent $m$ that is a manifestation of multifractality of the distribution of eddies (Arimitsu and Arimitsu, 2000a) as well as of scaling of the velocity structure functions (Arimitsu and Arimitsu, 2001). Moreover, the context of generalized thermo-statistics provides analytical formulas for PDFs of distance dependent velocity differences, where from the cascade like structure of the turbulent dynamics also an interpretation of the parameter $\kappa=1 /(q-1)$ is suggested (Beck, 2000). We relate in the following nonlocality in turbulent flows to the presence of long-range forces in nonextensive systems and demonstrate in the context of entropy generalization the consistency of the theoretical bi-kappa distribution (Leubner, 2004a) with observed, scale dependent PDFs of characteristic variables in the intermittent, turbulent interplanetary medium, where $\kappa$ appears as the only tuning parameter between the scales.

\section{Theory}

The nonextensive entropy generalization for the classical thermo-statistics proposed by Tsallis (1988) takes the form

$S_{q}=k_{B} \frac{1-\sum p_{i}^{q}}{q-1}$,

where $p_{i}$ is the probability of the $i$-th microstate, $k_{B}$ is Boltzmann's constant and $q$ is a parameter quantifying the degree of nonextensivity of the system, commonly referred to as the entropic index. A crucial property of this entropy is the pseudo-additivity such that

$S_{q}(A+B)=S_{q}(A)+S_{q}(B)+(1-q) S_{q}(A) S_{q}(B)$

for given subsystems $A$ and $B$ in the sense of factorizability of the microstate probabilities. Hence, nonlocality or long-range interactions are introduced by the multiplicative term accounting for correlations between the subsystems. In order to link the Tsallis q-statistics to the family of $\kappa$ distributions applied in space and astrophysical plasma modeling, we perform the transformation $1 /(1-q)=\kappa$ to Eq. (1) yielding the generalized entropy of the form (Leubner, 2002)

$S_{\kappa}=\kappa k_{B}\left(\sum p_{i}^{1-1 / \kappa}-1\right)$, 
where $\kappa=\infty$ corresponds to $q=1$ and represents the extensive limit of statistical independency. Consequently the interaction term in Eq. (2) cancels and the classical BoltzmannGibbs-Shannon entropy $S_{q}=-k_{B} \sum p_{i} \ln p_{i}$ is recovered.

Equation (2) applies to systems subject to long-range interactions or memory and systems evolving in a non-Euclidean and multifractal space-time. A further generalization of Eq. (2) for complex systems, composed of an arbitrary number of mutually correlated systems, is provided by Milovanov and Zelenyi (2000), where appropriate higher order terms in the entropy appear. Once the entropy is known the corresponding probability distributions are available.

In Maxwells derivation the velocity components of the distribution $f(v)$ are uncorrelated where $\ln f$ can be expressed as a sum of the logarithms of the one dimensional distribution functions. In nonextensive systems one needs to introduce correlations between the components accounting for the long-range interactions, which is conveniently done replacing the logarithm functions by a power law. Upon transforming the " $q-e x p$ " and " $q-\log$ " functions introduced in nonextensive statistics (Silva et al., 1998) into the $\kappa$ equivalents $e_{\kappa}(f)=[1+f / \kappa]^{\kappa}$ and $\ln \kappa(f)=\kappa\left[f^{1 / \kappa}-1\right]$ the generalized nonextensive solution for the one-dimensional distribution in $\kappa$ notation reads

$f_{h}(v)=\frac{N}{\sqrt{\pi} v_{t}} \frac{1}{\sqrt{\kappa}} \frac{\Gamma[\kappa]}{\Gamma[\kappa-1 / 2]}\left[1+\frac{1}{\kappa} \frac{v^{2}}{v_{t}^{2}}\right]^{-\kappa}$.

Hence, the exponential probability function of the Maxwellian gas of an uncorrelated ensemble of particles is replaced by the characteristics of a power law. We assign to $f_{h}$ the notation "halo" distribution where the Maxwellian normalization to the particle density $N$ is modified by a function of $\kappa . \quad v_{t}=\sqrt{2 k_{B} T / m}$ is the thermal speed of a Maxwellian, where $T$ and $m$ are the temperature and mass, respectively, of the species considered. The case $\kappa=\infty$ recovers the Maxwellian equilibrium distribution, see Fig. 1 and the discussion below.

We note that contrary to Eq. (4), derived from the nonextensive entropy generalization Eq. (3), the functional form of the $\kappa$-distributions conventionally used in plasma physics is powered by $-\kappa+1$ instead of $-\kappa$ (Leubner, 2002, 2004a). Consequently, the nonextensive distribution (4) provides more pronounced tails as compared to the conventional $\kappa$ distribution for the same values of $\kappa$. Furthermore, in all space plasma applications the spectral index $\kappa$ was hitherto assumed to be restricted to positive values, requiring in the derived one-dimensional nonextensive representation $3 / 2<\kappa \leq \infty$ equivalent to $-1<q \leq 1$.

Since the nonextensive formalism is not restricted to $-1<q \leq 1$ we generalize to values $q>1$ providing the full range $-\infty \leq \kappa \leq \infty$ for the spectral index $\kappa$. Hence, upon incorporating the sign into the equation, the one-dimensional distribution for negative definite $\kappa$ can be written in the form

$f_{c}(v)=\frac{N}{\sqrt{\pi} v_{t}} \frac{1}{\sqrt{\kappa}} \frac{\Gamma[\kappa+3 / 2]}{\Gamma[\kappa+1]}\left[1-\frac{1}{\kappa} \frac{v^{2}}{v_{t}^{2}}\right]^{\kappa}$.

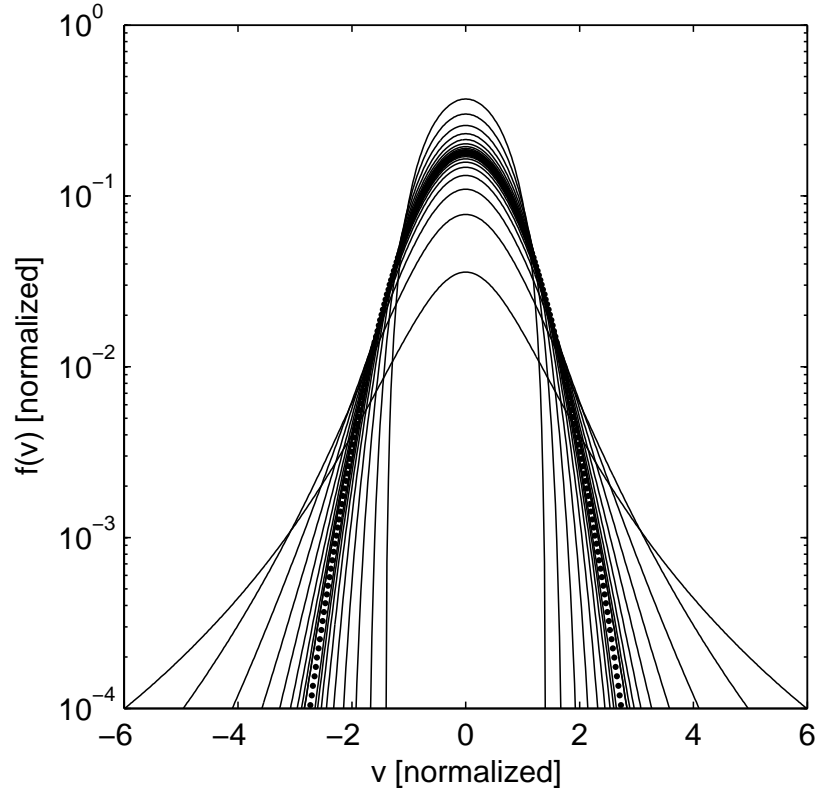

Fig. 1. A schematic plot of the characteristics of the nonextensive bi-kappa distribution family Eq. (6): with $\kappa=3$ the outermost and innermost curve correspond to the halo $f_{h}$ and core $f_{c}$ distribution fraction. For increasing $\kappa$-values both sets of curves merge at the same Maxwellian limit, indicated as bold line, $f_{h}$ from outside and $f_{c}$ from inside.

We assign the notation "core" distribution to $f_{c}$, a function subject to a thermal cutoff at the maximum allowed velocity $v_{\max }=\sqrt{\kappa} v_{t}$, which limits also the required integrations. Since the sign is implemented into Eq. (5), $\kappa$ has to be understood as a positive quantity wherever it appears. The nonextensive distribution-functionals (4) and (5) provide with the proper normalization from the level of entropy generalization Eq. (3) access to nonlocal effects due to the inclusion of longrange forces where $\kappa$ measures the degree of nonextensivity or mixing within the system.

Calculating the second moments of the one-dimensional distribution function (4) yields as consequence of the suprathermal tail structure a $\kappa$ dependent enhancement of the generalized temperature and corresponds to an entropy increase within the system (Leubner, 2004a). The second moment of the distribution function (5) provides the corresponding reduced generalized thermal speeds caused by the cutoff in velocity space, which results in an entropy decrease within the system due to the evolution of a higher organized state as $\kappa$ decreases (Leubner, 2004a). Both functions, $f_{h}$ and $f_{c}$ in Eqs. (4) and (5) approach the same Maxwellian as $\kappa \rightarrow \infty$. Figure 1 demonstrates schematically the non-thermal behavior of both, the suprathermal halo component $f_{h}$ and the reduced core distribution $f_{c}$, subject to finite support in velocity space and exhibiting a thermal cutoff at $v_{\max }=\sqrt{\kappa} v_{t}$.

It should be emphasized that, besides a brief comment by Leubner (2002) the extension to negative $\kappa$-values, as formulated in Eq. (5), was only recently recognized as being 
physically relevant (Leubner, 2004a,b), since research focused hitherto in particular on the suprathermal particle populations, well represented by a halo distribution. Let us point out that the thermal cutoff of the functional Eq. (5) provides apparently a clear definition of a core population, whereas a "core" of the function $f_{h}$ is not definable and just in some arbitrary way related to the central part of a Maxwellian. Besides a clear defined core-halo transition any unique and physically relevant nonextensive PDF must be subject to three further conditions. We require that (a) the distribution approaches one and the same Maxwellian as $\kappa \rightarrow \infty$, (b) a unique, global distribution must be definable by one single density and temperature and (c) upon variation of the coupling parameter $\kappa$ particle conservation and adiabatic evolution (Baumjohann and Paschmann, 1989) should be considered, such that a redistribution in a box (a source free environment) can be performed. Subject to these constraints the appropriate mathematical functional, representing observed core-halo structures in nonextensive astrophysical environments, is available from the elementary combination $f_{c h}=B_{c h}\left(f_{h}+f_{c}\right)$. In this context the full velocity space distribution, compatible with nonextensive entropy generalization and obeying the above constraints, reads

$$
F_{c h}(v ; \kappa)=\frac{N}{\pi^{1 / 2} v_{t}} G(\kappa)\left\{\left[1+\frac{1}{\kappa} \frac{v^{2}}{v_{t}^{2}}\right]^{-\kappa}+\left[1-\frac{1}{\kappa} \frac{v^{2}}{v_{t}^{2}}\right]^{\kappa}\right\}
$$

to which we assign the notation "bi-kappa distribution" and where $G(\kappa)$ is defined by

$G(\kappa)=\left[\frac{\kappa^{1 / 2} \Gamma(\kappa-1 / 2)}{\Gamma(\kappa)}+\frac{\kappa^{1 / 2} \Gamma(\kappa+1)}{\Gamma(\kappa+3 / 2)}\right]^{-1}$

from the normalization. The function $G(\kappa)$ is subject to a particular weak $\kappa$-dependence where $G(\kappa) \sim 1 / 2$, see Leubner (2004a) for a graphical illustration and discussion. Hence, the resulting factor $1 / 2$ in the normalization constant reflects the superposition of the two counter-organizing contributions in Eq. (6). Upon re-distribution of particles as consequence of long-range interactions, where $\kappa$ act as measure of nonlocality in the system, a fraction of coreparticles is removed and available to generate a pronounced non-Maxwellian tail population counting for zero net particle budget. Hence, the specific nonextensive velocity space distribution (6) provides a unique mathematical core-halo representation subject to the non-trivial property of particle conservation in the sense that the normalization is independent of the parameter $\kappa$ (Leubner, 2004a,b). This specific feature allows also to transform the one-dimensional bi-kappa velocity distribution of Maxwell's particle context into the mathematical form of a Gaussian of constant variance, a PDF applicable to any characteristic physical variable suitable to analyze statistical properties of astrophysical systems, see below. Contrary to the conventional $\kappa$-distribution models constraint by a positive $\kappa$-value, the functional form (6) permits in a closed system a re-distribution of energy from a Maxwellian into highly non-thermal core-halo structures where no support from the environment is required. This particular physical property is only available if $\kappa$ assumes the same value for both, core and halo fraction and is manifest simultaneously in a core-width reduction and distinct tail formation as $\kappa$ decreases. For $\kappa=\infty$ and $G(\kappa)=1 / 2$ the power laws in the brackets of the right hand side of Eq. (6) turn each into the same Maxwellian exponential.

The probability distribution functions of the Tsallis ensemble are linked to the analysis of fully developed turbulence (Arimitsu and Arimitsu, 2000a, 2001), where the nonextensive parameter $q$ is in particular related to nonlocality in the system and measures the scale dependence of the PDFs of the dynamical physical variables considered. In this conjunction it was also shown that the value of the index $q$ of nonextensive statistics is related to the extremes of the corresponding multifractal spectrum (Lyra and Tsallis, 1998).

We analyze in the following intermittency in astrophysical plasma turbulence by comparing observed PDFs of fluctuations of the velocity field magnitude in the solar wind with the global theoretical core-halo representation (6) accounting for nonlocality and long-range forces through entropy generalization. Furthermore, on small scales also the PDFs of interplanetary density and magnetic field fluctuations are observed to obey the same leptokurtic core non-Gaussianity along with pronounced tails or halos, requiring a generalization of Eq. (6). The Castaing approach, which models an energy cascade process via a convolution of Gaussians of different variances, is widely introduced to study intermittency in astrophysical plasma environments with regard to different characteristic physical variables. However, although most popular, the Castaing context provides no justification for nonlocality and long-range interactions in turbulence, though in its general form it is not restricted to the description of cascades. This fact requires to establish a unique, global PDF for any physical variable, provided in the context of nonextensive thermo-statistics, where nonlocality is introduced by pseudo-additive entropy generalization. Upon normalizing the one-dimensional bi-kappa particle distribution (6) to unity and assigning a unique distribution variance $\sigma$ to the thermal spread $v_{t}$ the "Maxwellian form" of the bi-kappa distribution (6) transforms to a "Gaussian form" of a global bi-kappa PDF as

$$
P_{c h}(\delta X ; \kappa)=\frac{1}{2 \sqrt{\pi} \sigma}\left\{\left[1+\frac{\delta X^{2}}{\kappa \sigma^{2}}\right]^{-\kappa}+\left[1-\frac{\delta X^{2}}{\kappa \sigma^{2}}\right]^{\kappa}\right\} .
$$

Subject to a single constant variance $\sigma$ this one-parameter PDF is applicable to the differences of the fluctuations $\delta X(\tau)=X(t+\tau)-X(t)$ of any physical variable $X$ in the astrophysical system considered. Here $\kappa$ assumes a clear physical interpretation defining the degree of nonextensivity or nonlocality in the system, thus being a measure of long-range interactions. As $\kappa \rightarrow \infty$ the bi-kappa distribution $P_{c h}(\delta X ; \kappa)$ approaches a single Gaussian. We proof in the following the relevance of the nonextensive, global bi-kappa PDF (8) on 
the observed scale dependence of the PDFs of the differences of magnetic field and velocity magnitude variables in the intermittent, turbulent interplanetary medium.

\section{Data evaluation}

For a proper estimation of empirical PDFs of interplanetary plasma and magnetic field data long sets are needed. Due to non-stationarity in driving-dissipation conditions, however, the data sets have to be chosen carefully. For the present approach solar wind velocity data are analyzed with a time resolution of $92 \mathrm{sec}$ available from the WIND spacecraft. WIND has a complicated trajectory that also crosses the magnetosphere from time to time. Our analysis is based on the entire year 1997 data set when WIND was in the GSE positions 150-230 RE. This WIND period is compared by a shorter time period of 10-29 January 1998 when magnetic field measurements with time resolution of $16 \mathrm{~s}$ were available from the ACE spacecraft. The ACE satellite is continuously monitoring the solar wind at the L1 point. Data gaps were interpolated in all cases and the resulting PDFs are fitted by the theoretical nonextensive distributions. In particular, from the 1997 WIND velocity field data 5 day periods of low speed subintervals were selected such that during each subinterval the bulk velocity was less than $450 \mathrm{~km} / \mathrm{s}$.

No velocity observations were available from ACE during the interval of 10-29 January 1998. However, at this time WIND was upstream, close to the ACE position. WIND measurements provide clear evidence that between 10-29 January 1998 the bulk speed was less than $450 \mathrm{~km} / \mathrm{s}$ in about $92 \%$ of this period. This interval was aslo subdivided to shorter subintervals to be able to compute the standard deviations.

For each data set the plasma and magnetic field increments were calculated at a given time lag $\tau$, equivalent to a particular spatial scale by $\delta X(\tau)=X(t+\tau)-X(t)$, where any variable within each subinterval is normalized to the standard deviation. For each realization the empirical probability distribution function (histogram) was then computed. $\delta X(\tau)$ is binned into $N$ equal spaced boxes and the number of elements in each box was computed where the robustness of the histograms against $N$ is tested. $\delta X(\tau)$ represents characteristic fluctuations at the time scale $\tau$ or, equivalently, across eddies of size $l \sim v \tau$. In case of single spacecraft measurements a similarity in statistical properties of temporal or spatial fluctuations is supposed. This is true in particular for the solar wind (Marsch and Tu, 1997), where the spatial fluctuations on a scale $l$ pass over the spacecraft faster than they typically fluctuate in time. Hence, by changing $\tau$ it is possible to analyze the statistical features of fluctuations in different time scales, which roughly correspond to those statistical characteristics across turbulent eddies of size $l \sim v \tau$.

At large scales, e.g. $\tau=24 \mathrm{~h}$ and $l \sim 5000 R E$ (supposing $v=350 \mathrm{~km} / \mathrm{s}$ ) the PDF's are close to the Gaussian distribution, because the correlation between the separated points vanishes, whereas at small scales, e.g. $\tau=0.2 \mathrm{~h}$ and $l \sim 40 \mathrm{RE}$ the PDF's have clearly non-Gaussian shapes, see Sect. 4, values estimated also by Sorriso-Valvo et al. (1999).

\section{Discussion and summary}

The Wind and ACE solar wind data analysis unambiguously manifests that the PDFs of large scale velocity and magnetic field fluctuations are well represented by a Gaussian, turning into leptokurtic peaked distributions of strong nonGaussianity in the center along with a pronounced tail structure at smaller scales. In particular, the PDFs of large-scale magnetic field fluctuations, not related to the increment field are known to be subject to relatively small deviations from the Gaussian statistics and are well fitted by the Castaing distribution, a convolution of Gaussians with variances distributed according to a log-normal distribution (Castaing et al., 1990; Padhye et al., 2001). Assuming a constant energy transfer rate between spatial scales all quantities exhibit a Gaussian distribution of fluctuations in this context. Independent of the physical situation considered, the Castaing distribution provides a multi-parameter description of observed PDFs, plausible in this case, since the large-scale fluctuations of the interplanetary magnetic field are generated by a variety of discrete coronal sources. If individual coronal sources evoke Gaussian distributed magnetic fields, the net magnetic fluctuations can be modeled by their superposition with a spread of the corresponding variances.

Contrary, small-scale fluctuations are associated with local intermittent flows where fluctuations are concentrated in limited space volumes. Consequently, the PDFs are scale dependent and intermittency generates long-tailed distributions. It is customary to use $n$-th order absolute powers of the plasma variables and magnetic field increments ( $n$-th order structure functions (Pagel and Balogh, 2001; Marsch and Tu, 1997) allowing to investigate the multi-scale scaling features of fluctuations subject to long-tailed PDFs. Direct studies of observed PDFs of the increment fields $\delta X(\tau)=X(t+\tau)-X(t)$ for any characteristic solar wind variable at time $t$ and time lag $\tau$ revealed departures from a Gaussian distribution over multiple scales (Sorriso-Valvo et al., 1999) and an increase of intermittency towards small scales (Marsch and Tu, 1994). The PDFs are also found to be leptokurtic, which indicates the turbulent character of the underlying fluctuations. Sorriso-Valvo et al. (1999) have shown that the non-Gaussian behavior of small-scale velocity and magnetic field fluctuations in the solar wind can also be described well by a Castaing distribution where the individual sources of Gaussian fluctuations appear at small-scales in turbulent cascades.

Here we demonstrate by means of Eqs. (6) or (8), respectively, from first principle statistics that the strong non-Gaussianity of the PDF of small scale fluctuations should be associated physically with long-range interactions provided in nonextensive systems by pseudo-additive entropy generalization. The scale dependence of the PDF in the solar wind is accurately represented via the only tuning parameter $\kappa$ of the bi-kappa distribution, 

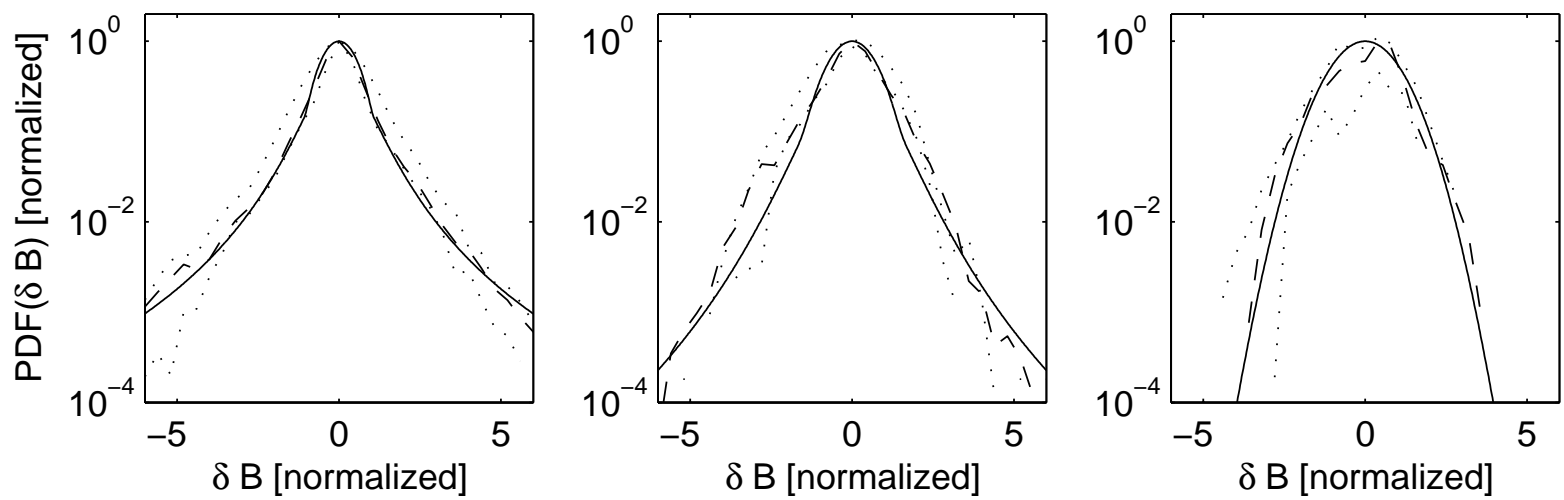

Fig. 2. The PDF of the increments of observed ACE magnetic field fluctuations for $\tau=100$ and a resolution of $16 \mathrm{~s}$ as compared to the bi-kappa function with $\kappa=1.8$. Based on the same data the central panel provides the characteristics for increased $\tau=2000$ where $\kappa$ assumes a value of 3.0 for the best representation. The PDF of large-scale magnetic field fluctuations, $\tau=10000$, are well modeled by a Gaussian with $\kappa=\infty$, right panel. The dotted lines correspond to the standard deviations.
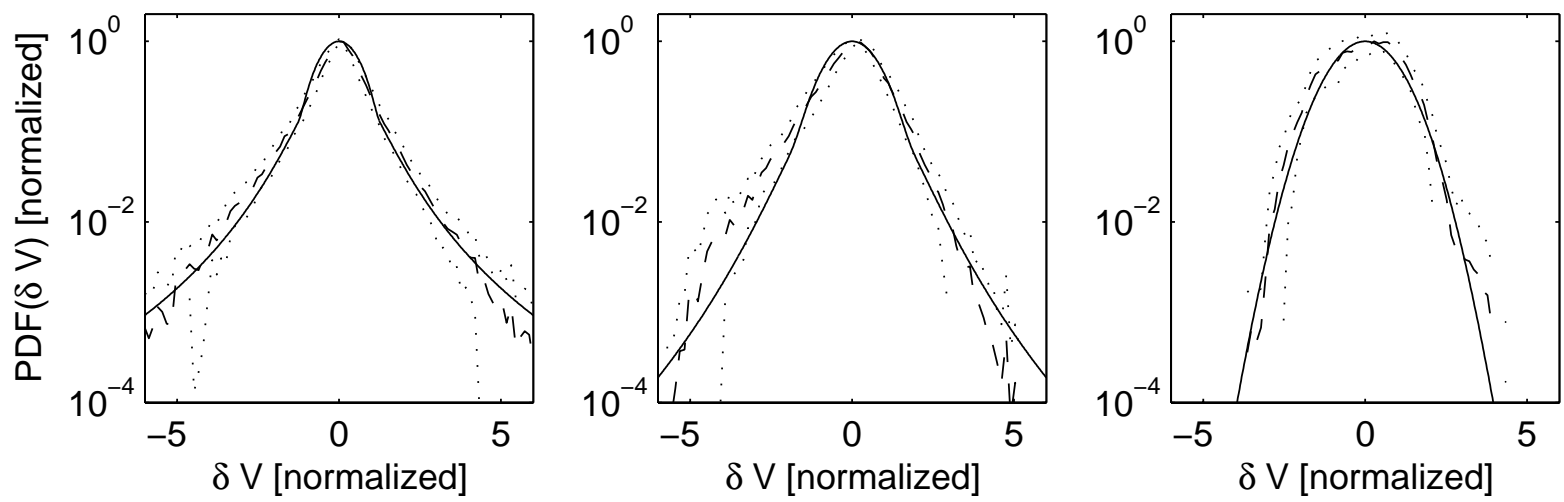

Fig. 3. The PDF of the increments of observed WIND velocity field magnitude fluctuations for $\tau=10$ and a resolution of $92 \mathrm{~s}$ as compared to the bi-kappa function with $\kappa=2$. Based on the same data the central panel provides the characteristics for increased $\tau=70$ where $\kappa$ assumes a value of 3.5 for the best representation. The PDF of large-scale velocity fluctuations are well modeled by a Gaussian with $\kappa=\infty$, right panel. The dotted lines correspond to the standard deviations.

measuring the degree of nonextensivity or coupling within the astrophysical plasma environment. As an example, in Fig. 2 undisturbed solar wind ACE magnetic field amplitude data of $16 \mathrm{~s}$ time resolution are analyzed where the dimensionless $\tau$ is multiplied by the resolution to generate an effective time-lag. In particular, the scale dependent PDF evolution of magnetic field fluctuations is subject to a two point separation scale of $\tau=100,2000$, and 10000. The corresponding best fits of the bi-kappa distribution are obtained for $\kappa=1.8,3$, and $\infty$, measuring the degree of nonextensivity, or coupling, respectively, through long-range interactions and the dotted lines refer to the standard deviation. The highly significant accuracy of the bi-kappa distribution fit demonstrates that non-locality in turbulence, when introduced theoretically by long-range interactions through the nonextensive context, generates a precise representation for the observed PDFs characterizing the intermittency of the fluctuations at all scales.
Based on WIND velocity field magnitude data Fig. 3 presents an analysis of the scale dependence of interplanetary PDFs of the velocity field magnitude showing in three plots from left to right the decreasing intermittency with increasing spatial separation scale or time-lags, where the observational uncertainty is again indicated by the standard deviation (dotted lines). Constraint by a time resolution of $92 \mathrm{~s}$ the two point time separation is $\tau=92 \mathrm{~s}$ where $\tau$ assumes the values 10,70 and 900 from left to right. The solid lines represent best fits to the observed PDFs with nonextensive distributions, where the corresponding $\kappa$ is determined as $\kappa=2,3.5$ and $\infty$. The strong non-Gaussian character of the eptokurtic PDFs (left panel), exhibiting pronounced tails associated with solar wind turbulence and intermittency in smallscale fluctuations, finds again an accurate analytical fit and hence a physical background in the nonextensive representation, accounting for nonlocality in turbulence via the longrange forces. The non-Gaussian structure is somewhat softened for enhanced $\tau=70$ (central panel) but again precisely 
modeled within the pseudo-additive entropy context, turning into the Gaussian shape of large scale fluctuations, which is independent of the increment field.

From the corresponding nonextensive WIND data analysis of the density and magnetic field fluctuations (Leubner and Vörös, 2004c) it is evident that the scale dependent characteristics of the observed PDFs of the increment fields $\delta X(\tau)=X(t+\tau)-X(t)$ for solar wind variables evolve simultaneously on small scales, approaching independency of the increment field in the large- scale Gaussian. Highly accurately, the overall scale dependence appears as a general characteristic of quiet astrophysical plasma environments indicating a universal scaling dependency between density, velocity and magnetic field intermittency within the experimental uncertainties. This strong correlation implies that the scale dependencies of all physical variables are coupled, where the solar wind Alfvénic fluctuations provide a physical basis of the velocity and magnetic field correlations. On the other hand, according to recent analyses, the magnetic field intensity exhibits a higher degree of intermittency than the solar wind bulk velocity, both in fast and slow winds (SorrisoValvo et al., 2001). However, Veltri and Mangeney (1999) found that the most intermittent structures in the slow wind are shock waves, displaying similar intermittency in the magnetic field intensity and bulk velocity. Furthermore, the proportionality between density fluctuations and the magnetic field and velocity fluctuations is already maintained in the solar wind by the presence of weak spatial gradients (Spangler, 2003).

Finally, Fig. 4 provides an estimation of the functional dependence between the time lag $\tau$ or spatial separation scale and the nonextensive parameter $\kappa$ for best fitting bi-kappa functions to the observed PDFs. As $\tau$ increases from small scales to the intermediate regime a pronounced plateau formation is established, i.e. the relative increase in $\kappa$-values with enhanced scales appears reduced. Such a behavior may indicate the presence of a transitional dynamical element characterizing a balance between long and short-range interactions. However, finite size effects can also influence the estimated functional evolution of the nonextensive parameter on the scale dependence, a subject to be analyzed in detail.

$\kappa$-distributions reproduce the Maxwell-Boltzmann distribution for $\kappa \rightarrow \infty$, a situation identifying $\kappa$ as an ordering parameter that acounts for correlations within the system. Quasistationary, highly correlated turbulent conditions characterized by kappa distributions represent stationary states far from equilibrium where a generalization of the BoltzmannShannon entropy, as measure of the level of organization or intermittency, applies (Goldstein and Lebowitz, 2004; Treumann, 2004). Physically this can be understood considering a system at a certain nonlinear stage where turbulence may reach a state of high energy level that is balanced by turbulent dissipation. In this environment equilibrium statistics can be extended to dissipative systems, approaching a stationary state beyond thermal equilibrium (Gotoh and Kraichnan, 2002). Since turbulence is driven in the solar wind by velocity shears we have choosen for the data

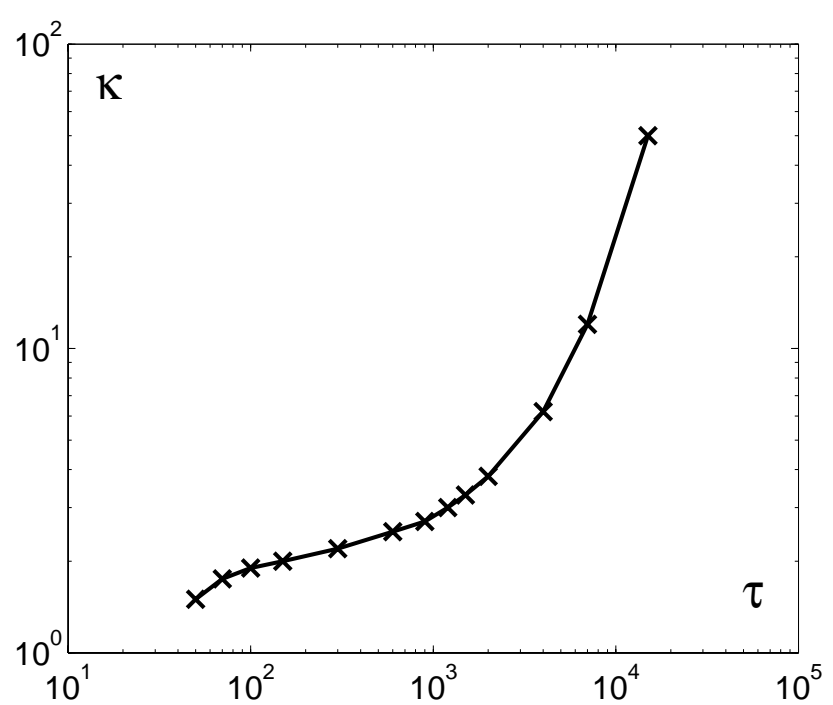

Fig. 4. The functional dependence of the spatial scale $\tau(\kappa)$.

analysis intervals of low speed solar wind with limits in velocity space, where the driving and dissipation conditions do not change significantly, maintaining therefore the dynamical equilibrium condition.

An analysis of fully developed turbulence in terms of Tsallis statistics, comparing scaling exponents of velocity structure functions of different models including the log-normal type, strongly indicates that the underlying statistics is that of the Tsallis ensemble and hence of a $\kappa$-distribution representation (Arimitsu and Arimitsu, 2000a,b). Moreover, the nonextensive character of turbulent dynamics is further supported relating the excess turbulent entropy to the pseudoadditive property (Anselmet et al., 1984). Arimitsu and Arimitsu (2001) calculated the extreme of the Tsallis entropy associated with multifractal dissipation structures of cascading turbulent energy and derived the corresponding velocity structure functions analytically. They compared the scaling exponents of the $m$-th order velocity structure functions from the generalized statistics model with the experimental results of Anselmet et al. (1984) and Meneveau and Sreenivasan (1991) and found that the scaling exponents are consistent with experimental data. Other models such as the Kolmogorov, the beta and the log-normal models showed large deviations from experimental data for $m>10$.

A multi-scale cascade mechanism is not the only way for a realization of long range interactions. Let us provide a physical situation where large and small scales are directly coupled and the nonlocal energy transfer is not induced by cascading processes, following e.g. a log-normal model (Frisch, 1996).

As an example consider the nonlinear terms in NavierStokes equations containing the vector product of vorticity $(\boldsymbol{\omega})$ and velocity $(\mathbf{V})$ fields. In turbulent flows the correlations between vorticity and velocity fields are close to zero. Nevertheless, bi-directional coupling between the large scales (velocity field) and small scales (vorticity field) is unavoidable 
without cascades. In this picture any changes in the vorticity small-scale field are reacting back to the large-scale velocity field through $\boldsymbol{\omega}=\operatorname{curl} \mathbf{V}$ (Tsinober, 1990) where vorticity is not restricted to small scales only. The large-scale velocity field is entirely determined by the specific boundary conditions and vorticity through $\nabla^{2} \mathbf{V}=-$ curl $\omega$, which includes the velocity field itself, indicating that the direct interaction is bi-directional (Tsinober, 2001).

A further example is found in the context of MHD where Chang (1999) proposed an intermittent turbulence model for the solar wind and for the Earth's magnetotail, which comprises neither cascades nor requires local interactions in Fourier space. In this scenario non-propagating or convected fluctuations generate multiscale coherent structures (e.g. flux tubes), which can interact, deform and produce new sites of non-propagating fluctuations. Coherent structures of the same polarity merge into a structure with lower local energetic state, while structures of opposite polarities may repel each other. This coherent structures can be considered as discrete interacting "particles" in MHD flows, responsible for the particular entropy within the system and validating the analogy to the kinetic level of PDFs. Chang et al. (2004) have computed PDFs of the intermittent fluctuations from direct numerical simulations of interacting coherent structures. The resulting PDFs have typical leptokurtic shapes, which can be well fitted again by a variety of models, including those with predominantly local interactions in Fourier space. Since all models provide similar fitting accuaracy it is required to focus on the underlying physical situation in turbulent flows. Hence, with regard to nonlocal interactions not based on cascade processes the nonextensive entropy approach provides physically a justification for nonlocal interactions and should therefore be favored over cascade models in such processes.

On observational grounds in the solar wind nonpropagating fluctuations perpendicular to the mean magnetic field are energetically dominant. The energy partitioning between nonpropagating fluctuations and waves is about $80 \%-$ $20 \%$ (e.g. (Bieber et al., 1996)). Non-propagating perpendicular fluctuations can be generated also by opposite traveling wave packets, producing perpendicular cascades in Fourier space (Shebalin et al., 1983). Therefore MHD turbulence in the solar wind is generally in a state in which propagating waves and non-propagating fluctuations coexist. Moreover, typical processes include fluctuation induced multiscale interactions of coherent structures for which a local interaction in Fourier space is not required and interactions of opposite traveling waves are followed by energy cascades in Fourier space. Physically, at least a part of the nonlocal forces and turbulent intermittency results from a source exhibiting nonlocal interactions in the Fourier space. Since the coherent structures or flux tubes merge and interact (Chang et al., 2004) they may correlate over long distances, leading to topological complexity of the underlying structures (Consolini, 2002).

The entropy quantifies the degree of structuring in intermittent turbulence expressed through singular multifractal measures, where also the parameter $\kappa$ (or $q$ ) is related to the extremes of multifractal distributions (Lyra and Tsallis, 1998; Arimitsu and Arimitsu, 2000a,b; Beck, 2000). Since the nonextensive entropy approach is independent of the mechanism leading to the structures - in both situations, cascading processes and multiscale interacting coherent structures - or even in coexisting situations, the entropy concept can be applied for the analysis and quantification of resulting characteristics in turbulence. In summary, the majority of hitherto existing models of intermittency in the solar wind essentially correspond to the cascade picture of turbulence. Small-scale intermittency, however, can be associated also by emerging topological complexity of coherent sturctures in turbulence, which might be understood better through entropy concepts, disregarding the goodness criteria of different fits.

We provided specific examples based on multiscale interacting coherent structures where the traditional cascade and thus e.g. the log-normal approach beside others - should not be applied for physical reasons (not in terms of PDF fitting accuracy). Therefore the proposed context based on entropy ganeralization has a potential to describe the underlying physics suitably and thus justifies the nonextensive approach. Certainly this must be viewed as an incomplete concept in view of the complexity of intermittence in turbulence (Cohen, 2004).

The PDFs of fluctuations in the intermittent interplanetary medium are equaly well represented by different fits provided for instance by the Castaing, multifractal, log-normal or shell model. However, turbulence is a multiscale process subject to long-range forces where the different models are degenerate in the sense that they are equally well characterizing intermittency. The above models introduce nonlocal interactions between separated scales through an energy cascade via a fragmentation process, resulting in long-range correlations. Therefore, the log-normal or the shell-model contain a priori assumptions about the nature of long-range forces. Using these models in statistical experimental studies to obtain the spatial dependence and characteristics of nonlocal forces, the outcome mimics long-range correlations in a cascade process because of the a priori assumptions built in. A multi-scale cascade mechanism is not the only way for a realisation of long-range interactions. Strong dissipation can occur within the inertial range due to a concurrent physical process, or direct interaction between large and small scales is possible without any inertial range cascade. Consequently, we argue that the nonextensive context provides the most generic approach since long-range interactions and memory are introduced on the fundamental level of entropy generalization. There is no built in condition about the nature of the long-range forces or spatial dependence of the corresponding parameter $\kappa$. Since the nonextensive context is not restricted to any particular model it covers cascade processes as well. Thus, the nonextensive approach opens the possibility for experimental studies of specific mechanisms responsible for long-range interactions in turbulence without need to refer to a priori model assumptions. 
Summarizing, a bi-kappa distribution family turns out theoretically as consequence of the entropy generalization in nonextensive thermo-statistics and was successfully applied in velocity space to interplanetary core-halo electron distributions as well as double-humped proton structures. Upon transforming to the mathematical "Gaussian form" the particular global, one-parameter bi-kappa PDF of constant variance provides also access to the pronounced core-halo distributions observed in astrophysical plasma turbulence. A redistribution of a Gaussian on large scales can be performed in the interplanetary plasma, turning into highly non-Gaussian leptokurtic and long-tailed structures that are manifest on small scales. Pseudo-additive entropy generalization provides the required physical interpretation of the only tuning parameter $\kappa$ in terms of the degree of nonextensivity of the system as a measure of nonlocality or coupling due to longrange interactions. The scale dependence of the PDFs of the differences of characteristic physical variables in the intermittent turbulent interplanetary medium as velocity and magnetic field magnitude is accurately represented by the nonextensive bi-kappa functional. It is argued that the leptokurtic, long-tailed non-Gaussian core-halo PDFs characterizing the intermittency of the turbulent fluctuations must be related to the nonextensive character of the interplanetary medium accounting for long-range interaction via the entropy generalization.

Acknowledgements. M. Leubner acknowledges the hospitality of the Austrian Academy of Sciences at the Space Research Institute in Graz. The authors are grateful to N. Ness (Bartol Res. Inst.) for providing ACE data and to R. Lepping and K. W. Ogilvie (NASA-GSFC) for providing WIND data.

Edited by: P. Watkins

Reviewed by: one referee

\section{References}

Anselmet, F., Cagne, Y., Hopfinger, E. J., and Antonia, R. A.: High order velocity structure functions in turbulence shear flows, J. Fluid Mech., 140, 63-89, 1984.

Arimitsu, T. and Arimitsu, N.: Analysis of fully developed turbulence in terms of Tsallis statistics, Phys. Rev. E 61, 3237-3240, 2000a.

Arimitsu, T. and Arimitsu, N., Tsallis statistics and fully developed turbulence, J. Phys. A: Math. Gen., 33, L235-L241, 2000 b.

Arimitsu, T. and Arimitsu, N.: Analysis of fully developed turbulence by a generalized statistics, Prog. Theor. Phys., 105, 355360, 2001.

Baumjohann, W. and Paschmann, G.: Determination of the polytropic index in the plasma sheet, Geophys. Res. Lett., 16, 295298, 1989.

Beck, C.: Application of generalized thermostatistics to fully developed turbulence, Physica A, 277, 115-123, 2000.

Bieber, J. W., Wanner, W., and Matthaeus, W. H.: Dominant twodimensional solar wind turbulence with implications for cosmic ray transport. J. Geophys. Res., 101, 2511-2522, 1996.
Burlaga, L. F.: Multifractal structure of speed fluctuations in recurrent streams at $1 \mathrm{AU}$ and near $6 \mathrm{AU}$, Geophys. Res. Lett., 18, 1651-1654, 1991.

Burlaga, L. F.: Multifractal structure of the magnetic field and plasma in recurrent streams at 1 AU, J. Geophys. Res., 97, 42814293, 1992.

Burlaga, L. F., Richardson, J. D., and Wang, C.: Speed fluctuations near $60 \mathrm{AU}$ on scales from 1 day to 1 year: Observations and model, J. Geophys. Res., 107, A10, SSH20, 2002.

Burlaga, L. F.: Large-scale speed fluctuations at 1 AU on scales from 1 hour to 1 year: 1999 and 1995, J. Geophys. Res., 107, A11, SSH18, 2002.

Carbone, V., Veltri, P., and Bruno, R.: Experimental evidence for differences in the extended self-similarity scaling laws between fluid and magnetohydrodynamic turbulent flows, Phys. Rev. Lett., 75, 3110-3113, 1995.

Castaing, B. Y., Gagne, Y., and Hopfinger, E. J.: Velocity probability density functions of high Reynolds number turbulence, Physica D., 46, 177-200, 1990.

Castaing, B. Y. and Dubrulle, B.: Fully developed turbulence: A unifying point of view, J. Phys. II France 5, 895-899, 1995.

Castaing, B. Y.: The temperature of turbulent flows, J. Phys. II France 6, 105-114, 1996.

Chang, T.: Self-organized criticality, multifractal spectra, sporadic localized reconnections and intermittent turbulence in magnetotail, Phys. Plasmas, 6, 4137-4145, 1999.

Chang, T., Tam, S. W. Y., and Wu, Ch.: Complexity induced anisotropic bimodal intermittent turbulence in space plasmas, Phys. Plasmas, 11, 1287-1299, 2004.

Cohen, E. G. D.: Superstatistics, Physica D 193, 35-52, 2004.

Consolini, G. and De Michelis, P.: Non-Gaussian distribution function of AE-index fluctuations: Evidence for time intermittency, Geophys. Res. Lett., 25, 4087-4090, 1998.

Consolini, G. and Chang, T. S.: Complexity, magnetic field topology, criticality and metastability in magnetotail dynamics, J. Atm. Sol. Terr. Phys., 64, 541-549, 2002.

Frisch, U.: Turbulence, Cambridge University Press, Cambridge, 1996.

Goldstein, S. and Lebowitz, J. L.: On the (Boltzman) entropy of nonequilibrium systems, Physica D, 193, 53-66, 2004.

Gotoh, T. and Kraichnan, R. H.: Turbulence and Tsallis statistics, Physica D, 193, 231-244, 2004.

Guowei, He., Dubrulle, B., and Graner, F.: Thermodynamical versus log-Poisson distribution in turbulence, Phys. Lett. A 245, 419-424, 1998.

Hnat, B., Chapman, S. C., Rowlands G., Watkins, N. W., and Freeman, M. P.: Scaling of solar wind e and the AU, AL and AE indices as seen by WIND, Geophys. Res. Lett., 29, 2078-2082, doi:10.1029/2002GL016054, 2003.

Leubner, M. P.: A nonextensive entropy approach to kappadistributions, Astrophys. Space Sci., 282, 573-579, 2002.

Leubner, M. P.: Core-halo distribution functions - a natural equilibrium state in generalized thermo-statistics, Astrophys. J., 604, 469-478, 2004a.

Leubner, M. P.: Fundamental issued on kappa-distributions in space plasmas and interplanetary proton distributions, Phys. Plasmas, 11, 1308-1316, 2004b.

Leubner, M. P. and Vörös, Z.: A nonextensive entropy approach to solar wind intermittency, Astrophys. J., 618, 547-555, 2005.

Lyra, M. L. and Tsallis, C.: Nonextensivity and multifractality in low-dimensional dissipative systems, Phys. Rev. Lett., 80, 5356,1998 
Marsch, E. and Tu, C.-Y.: Non-Gaussian probability distributions of solar wind fluctuations, Ann. Geophys., 12, 1127-1138, 1994, SRef-ID: 1432-0576/ag/1994-12-1127.

Marsch, E. and Tu, C.-Y.: Intermittency, non-Gaussian statistics and fractal scaling of MHD fluctuations in the solar wind, Nonlin. Proc. Geophys., 4, 101-124, 1997,

\section{SRef-ID: 1607-7946/npg/1997-4-101.}

Matthaeus, W. H. and Goldstein, M. L.: Measurements of the rugged invariants of magnetohydrodynamic turbulence in the solar wind, J. Geophys. Res., 87, 6011-6028, 1982.

Meneveau, C. and Sreenivasan K. R.: The multifractal nature of turbulent energy dissipation, J. Fluid Mech., 224, 429-484, 1991.

Milovanov, A. V. and Zelenyi, L. M.: Functional background of the Tsallis entropy: 'coarse-grained' systems and 'kappa' functions, Nonlin. Proc. Geophys., 7, 211-221, 2000,

\section{SRef-ID: 1607-7946/npg/2000-7-211.}

Padhye, N. S., Smith, C. W., and Mathaeus, W. H.: Distribution of magnetic field components in the solar wind plasma, J. Geophys. Res., A106, 18 635-18 650, 2001.

Pagel, C. and Balogh, A.: A study of magnetic fluctuations and their anomalous scaling in the solar wind: the Ulysses fast-latitude scan, Nonlin. Proc. Geophys., 8, 313-330, 2001,

SRef-ID: 1607-7946/npg/2001-8-313.

Renyi, A.: On a new axiomatic theory of probability, Acta Math. Hungaria, 6, 285-335, 1955.

Schmitt, F. and Marsan, D.: Stochastic equations generating continuous multiplicative cascades, Eur. Phys. J., B 20, 3-6, 2001.

Shebalin, J. V., Matthaeus, W. H., and Montgomery, D. C.: Anisotropy in MHD turbulence due to a mean magnetic field, J. Plasma Phys., 29, 525-547, 1983.

Silva, R., Plastino, A. R., and Lima, J. A. S.: A Maxwellian path to q-nonextensive velocity distribution functions, Phys. Lett. A, 249, 401-408, 1998.

Sorriso-Valvo, L., Carbone, V., and Veltri, P.: Intermittency in the solar wind turbulence through probability distribution functions of fluctuations, Geophys. Res. Lett., 26, 1801-1804, 1999.

Sorriso-Valvo, L., Carbone, V., Giuliani, P., Veltri, P., Bruno, R., Antoni, V., and Martines, E.: Intermittency in plasma turbulence, Planet. Space Sci., 49, 1193-1200, 2001.
Spangler, S. R.: The small amplitude of density turbulence in the inner solar wind, Nonlin. Proc. Geophys., 21, 113-120, 2003.

Treumann, R. A.: Kinetic theoretical foundation of Lorentzian statistical mechanics, Physica Scripta 59, 19-26, 1999.

Treumann, R. A.: Generalized Lorentzian Thermodynamics, Physica Scripta 59, 204-214, 1999.

Treumann, R. A., Jaroschek, C. H., and Scholer, M.: Stationary plasma states far from equilibrium, Phys. Plasmas, 11, 13171325, 2004.

Tsallis, C.: Possible generalization of Boltzmann-Gibbs statistics, J. Stat. Phys., 52, 479-487, 1988.

Tsallis, C.: Non-extensive thermostatistics: brief review and comments, Physica A, 221, 277-290, 1995.

Tsinober, A.: On one property of Lamb vector in isotropic turbulent flow, Phys. Fluids, A2, 484-486, 1990.

Tsinober, A.: An informal introduction to turbulence, Kluwer Academic Publishers, Dodrecht, 2001.

Veltri, P. and Mangeney, A.: Scaling laws and intermittent structures in solar wind MHD turbulence, in: Solar Wind 9, edited by: Habbai, S. R., Hesser, R., Hollweg, J. V., and Isenberg, P. A., American Institute of Physics, 543-546, 1999.

Vörös, Z., Jankovičová, D., and Kovács, P.: Scaling and singularity characteristics of solar wind and magnetospheric fluctuations, Nonl. Proc. Geophys., 9, 149-162, 2002,

SRef-ID: 1607-7946/npg/2002-9-149.

Vörös, Z. and Jankovičová, D.: Neural network prediction of geomagnetic activity: a method using local Hölder exponents, Nonl Proc. Geophys., 9, 425-433, 2002,

SRef-ID: 1607-7946/npg/2002-9-425.

Vörös, Z., Baumjohann, W., Nakamura, R., et al.: Multi-scale magnetic field intermittence in the plasma sheet, Ann. Geophys., 21 1955-1964, 2003,

SRef-ID: 1432-0576/ag/2003-21-1955.

Vörös, Z., Baumjohann, W., Nakamura, R., et al.: Wavelet analysis of magnetic turbulence in the Earth's plasma sheet, Phys. Plasmas, 11, 1333-1338, 2004. 